\title{
Representações da relação entre exercício físico e saúde por pacientes fibromiálgicos
}

I ${ }^{1}$ Maria Lucia Alves Cavaliere, 2 Jerusa Mônica de Abreu Souza,

${ }^{3}$ José Silvio de Oliveira Barbosa I

Resumo: Fibromialgia (FM) é uma síndrome caracterizada por dor músculo esquelética crônica generalizada, fadiga, distúrbio do sono e sintomas associados. Preocupação com o controle da síndrome justifica-se por sua crescente prevalência e pelo impacto negativo na qualidade de vida dos afetados. Das opções de tratamento não-medicamentoso, o exercício físico (EF) é elemento terapêutico relevante. Há preocupação como o impacto da síndrome e sua terapêutica sobre a saúde, aqui entendida como um conjunto de dimensões - física, emocional, social, espiritual, intelectual e profissional - de uma pessoa. O objetivo do estudo foi identificar a percepção de pacientes com FM sobre a relação entre EF e saúde. Utilizou-se o referencial teórico-metodológico das Representações Sociais numa amostra de 22 mulheres de um projeto multidisciplinar: Educação Física, Nutrição, Medicina e Psicologia. A análise dos dados foi realizada a partir do conteúdo transcrito das gravações de entrevista semiestruturada e interpretação de ilustrações feitas pelas pacientes. Houve melhora na dimensão física, demonstrada pelo alívio da dor nas falas; houve também melhora na dimensão emocional, evidenciada pela melhora da autoestima indicada nas ilustraçôes. Com base na teoria utilizada, as pacientes reconhecem a relação positiva entre EF e saúde.
1 Técnico Educador Físico;

Mestrado; Instituto de Educação Física e Desportos, Universidade do Estado do Rio de Janeiro (UERJ). Endereços eletrônicos: : fibromialgialafisaef@gmail.com e malualves@hotmail.com

2 Técnico Educador Físico; Mestrado; Instituto de Educação Física e Desportos, UERJ.

${ }^{3}$ Professor assistente; Mestrado; Instituto de Educação Física e Desportos, UERJ. 


\section{Introdução}

A fibromialgia (FM) é uma síndrome caracterizada por dor músculo esquelética generalizada crônica, fadiga, distúrbio do sono e por uma multiplicidade de sintomas associados, inclusive de caráter psicopatol gico (WOLFE, 1990).

Em estudo de revisão, Cavalcante (2006) aponta prevalência da fibromialgia na população com valores entre 0,66 e 4,4\%, sendo significativamente mais frequente em mulheres, afetando adultos e crianças. Estudo realizado por Senna et al. (2004) em Montes Claros, Minas Gerais, Brasil, mostra que a FM foi a segunda desordem reumatológica mais freqüente, com prevalência de 2,5\%. A preocupação das entidades envolvidas no estudo e controle dessa síndrome justifica-se não só pela crescente prevalência, mas sobretudo pelo impacto negativo na qualidade de vida dos afetados (BENNETT, 1996; CATHEY, 1986; MARTINEZ, 1999; KAPLAN, 2000). Os pacientes podem ter sua capacidade de trabalho prejudicada (CATHEY, 1988; HAWLEY, 1988), apresentar capacidade reduzida de realizar exercícios físicos e baixo nível de aptidão cardiorrespiratória (BENNETT, 1989; MENGESHOEL, 1990; VALIM, 2002), o que pode comprometer as atividades de vida diária, com repercussôes psicossociais.

Ações multidisciplinares que incluem o tratamento farmacológico e nãofarmacológico são recomendadas para o controle da síndrome (PROVENZA, 2004; GOLDENBERG, 2004). E entre as opções não-medicamentosas, o exercício físico tem-se destacado como elemento terapêutico relevante em estudos clássicos realizados por McCain $(1986,1988)$ e em estudos mais recentes como observa Jones (2006) e Busch (2007).

Por se basear principalmente em aspectos de natureza clínica, certo grau de subjetividade marca a mensuração do estado de saúde dos pacientes. A avaliação da efetividade dos diferentes tratamentos para FM pode ficar comprometida, pois, além da subjetividade e variedade dos sintomas, há possibilidade de interferência de fatores físicos, psíquicos e ambientais na modulação dos sintomas. Dessa forma, algumas questôes ainda estão em aberto e a necessidade de esclarecimento mantém a busca por formas de avaliações objetivas. Assim, questionários de investigação sobre qualidade de vida avaliando grau de incapacidade física e estado global de saúde têm sido utilizados na tentativa de quantificar os efeitos das intervenções e de se aproximar da compreensão sobre questóes deste quadro de saúde. Considerando a definição de saúde da Organização Mundial da Saúde 
(WHO, 1978), que inclui o bem-estar físico, mental e social, é importante a

preocupação com a repercussão das doenças e das diversas medidas terapêuticas na vida dos pacientes na busca deste bem-estar.

Em estudo realizado em 2002, Cavaliere avaliou a efetividade de um programa multidisciplinar, que incluía exercícios físicos no tratamento da FM. Apesar de terem sido observadas melhoras na aptidão física (consumo máximo de oxigênio ${ }^{1}$ e flexibilidade ${ }^{2}$ ) e na vitalidade, item de qualidade de vida, não foram encontradas diferenças com significância estatística no período analisado com relação a alguns sintomas (intensidade da dor, número de pontos dolorosos, depressão), ao estado global de saúde, depressão e aos demais itens de qualidade de vida. Este resultado quantitativo foi diferente do relato apresentado pelas participantes, bem como pelas observações feitas pela equipe de tratamento.

O objetivo deste estudo foi identificar a percepção de pacientes com FM sobre a relação entre exercício físico e saúde. Desejava-se saber quais eram os conceitos e imagens da relação exercício físico e saúde que poderiam explicar a existência de expressiva melhora física, sem esperada repercussão no quadro clínico, e na qualidade de vida.

\section{Métodos}

Trata-se de estudo de natureza qualitativa e, neste sentido, é relevante considerar Spink (1995), quando ressalta que "a pesquisa de representações sociais", pelo tipo de situação estudada - "situação natural" em que se estabelece a "interação com as pessoas, em seus próprios territórios e através de sua própria linguagem” - é necessariamente uma pesquisa qualitativa.

Participaram deste estudo 22 mulheres usuárias de um programa de tratamento multidisciplinar para pacientes portadores de fibromialgia, realizado em uma universidade pública no Estado do Rio de Janeiro. O programa de tratamento constava de acompanhamento médico ambulatorial, treinamento físico oferecido em duas sessões semanais, em grupo, com intensidade de 70 a $85 \%$ da frequência cardíaca máxima obtida em teste ergométrico, sessões semanais de psicologia e nutrição em grupo e, quando necessário, atendimento individual. A idade das participantes variou ente 21 e 61 anos (média $=47,5$ anos e desvio padrão $=9,1$ ) e todas tinham diagnóstico de FM segundo os critérios estabelecidos pelo Colégio Americano de Reumatologia (WOLFE, 1990). Dentre as participantes, 12 
também tinham hipertensão arterial sistêmica. Dessa amostra, 14 particpavam há 12 meses de projeto, e oito, há 30 meses.

A coleta de dados foi realizada através de entrevista semiestruturada cuja questão introdutória foi: "Como você percebe, no seu dia a dia, a relação exercício físico e saúde?" As respostas foram gravadas em fita cassete e posteriormente transcritas. Também foi solicitado às participantes que expressassem sua percepção sobre a relação exercício físico e saúde sob a forma de uma ilustração. Assim seria possível detectar, segundo Souza Filho (1995), conteúdos latentes não revelados por levantamentos feitos com escalas de atitudes convencionais, nas quais é maior o controle racional do sujeito sobre suas respostas. Após a realização dos desenhos, as participantes verbalizaram a respeito de suas intenções e significados, sendo este momento também gravado e transcrito para ser analisado.

A análise dos dados foi realizada por dois avaliadores independentes, de forma separada, e posteriormente os resultados foram interpretados e comparados. As poucas discordâncias entre os avaliadores foram discutidas até o consenso.

Optou-se pela técnica de análise de conteúdo (BARDIN, 2000) para compreender, a partir dos relatos das entrevistadas, a percepção das participantes sobre a relação exercício físico e saúde. Assim, após a transcrição das fitas, foi feita uma leitura cuidadosa, entremeada sempre que preciso de nova escuta das entrevistas para se ter uma ideia global e posterior apreensão das falas identificadas como relevantes para a pesquisa. Foram elaboradas categorias específicas com base no conceito de saúde de Silva e De Marchi (1997), que se constitui de seis dimensôes: física, emocional, social, intelectual, espiritual e profissional.

A dimensão física envolve o quadro clínico do indivíduo, juntamente com práticas saudáveis e uso correto do sistema médico. A dimensão emocional considera desde o adequado gerenciamento de tensóes até a autoestima somados ao entusiasmo em relação à vida. A dimensão intelectual ilustra a capacidade criativa, a expansão de conhecimentos e o partilhamento do potencial interno. A dimensão social trata da qualidade de relacionamentos, harmonia familiar e com o meio ambiente. Dimensão espiritual é traduzida pelo propósito de vida associado a pensamentos positivos e otimistas. A dimensão profissional se refere a satisfação e reconhecimento do trabalho além de desenvolvimento profissional constante.

Este estudo foi seguimento da pesquisa "Efetividade de um programa multidisciplinar no tratamento da fibromialgia”, aprovada pelo Comitê de Ética em Pesquisa do Hospital Universitário Pedro Ernesto da Universidade do Estado 
do Rio de Janeiro (441-CEP/HUPE). Além disso, as participantes assinaram um termo de consentimento para participar da pesquisa (Anexo I).

\section{Resultados}

O quadro 1 mostra as representações encontradas na fala dos sujeitos com suas respectivas dimensões.

Quadro 1: Representaçóes e dimensóes das falas das participantes

\begin{tabular}{|l|c|}
\hline Representações & Dimensóes \\
\hline Alívio da dor $(\mathrm{n}=7)$ & Física \\
\hline Controle da dor $(\mathrm{n}=1)$ & Física \\
\hline Tolerância e convivência com a dor $(\mathrm{n}=6)$ & Física \\
\hline Retomada das atividades de vida diária $(\mathrm{n}=2)$ & Física \\
\hline Redução do cansaço $(\mathrm{n}=1)$ & Física \\
\hline Maior disposição física $(\mathrm{n}=4)$ & Física \\
\hline Estética ( $\mathrm{n}=1)$ & Física \\
\hline Aumento da auto-estima $(\mathrm{n}=2)$ & Emocional \\
\hline Redução da depressão $(\mathrm{n}=1)$ & Emocional \\
\hline Aumento da sensação de bem-estar $(\mathrm{n}=3)$ & Emocional \\
\hline Melhora do estado emocional $(\mathrm{n}=1)$ & Emocional \\
\hline Exercício como bom para saúde independente da dor $(\mathrm{n}=1)$ & Intelectual \\
\hline Aquisição de estilo de vida saudável independente da existência de doença $(\mathrm{n}=1)$ & Intelectual \\
\hline Identificação dos sintomas da FM (n=1) & Intelectual \\
\hline Reconhecimento da falta que o exercício físico faz $(\mathrm{n}=2)$ & Intelectual \\
\hline Reconhecimento dos limites $(\mathrm{n}=1)$ & Intelectual \\
\hline Educação postural ( $\mathrm{n}=1)$ & Intelectual \\
\hline Consciência corporal ( $\mathrm{n}=1)$ & Intelectual \\
\hline Convivência e motivação com o grupo $(\mathrm{n}=7)$ & Social \\
\hline Melhora do estado de espírito $(\mathrm{n}=1)$ & Espiritual \\
\hline
\end{tabular}

Cinco das seis dimensões propostas por Silva e De Marchi (1997) foram identificadas na análise das falas e das ilustraçôes das participantes: dimensão física, emocional, social, espiritual e intelectual. Apenas a dimensão profissional 
não foi mencionada. A dimensão mais ressaltada na análise das falas foi a física (100\%). Quando foi realizada a análise das falas referentes às ilustraçōes, a dimensão emocional foi a de maior representatividade $(77,27 \%)$.

O quadro 2 mostra as representações encontradas na análise das falas referentes às ilustrações e suas respectivas dimensões.

\section{Quadiro 2: Representaçóes e dimensóes das fallas referentes às illustraçóes}

\begin{tabular}{|l|c|}
\hline Representações & Dimensão \\
\hline Melhora da auto-estima $(\mathrm{n}=8)$ & Emocional \\
\hline Maior bem-estar $(\mathrm{n}=7)$ & Emocional \\
\hline Maior relaxamento $(\mathrm{n}=2)$ & Emocional \\
\hline Influência positiva do grupo $(\mathrm{n}=7)$ & Social \\
\hline Visão positiva da vida $(\mathrm{n}=5)$ & Espiritual \\
\hline Melhor condição física $(\mathrm{n}=1)$ & Física \\
\hline Estética ( $\mathrm{n}=1)$ & Física \\
\hline Melhor convivência com a dor $(\mathrm{n}=2)$ & Física \\
\hline Alívio da dor (n=1) & Física \\
\hline $\begin{array}{l}\text { Maior consciência em relação aos benefícios do } \\
\text { exercício físico sobre a saúde ( } \mathrm{n}=2)\end{array}$ & Intelectual \\
\hline $\begin{array}{l}\text { Consciência da relação do exercício físico e saúde independente } \\
\text { da existência de patologia }(\mathrm{n}=1)\end{array}$ & Intelectual \\
\hline
\end{tabular}

As falas e a figura abaixo são amostras aleatórias referentes a relação exercício físico e saúde que exemplificam as dimensões.

\section{Física}

[...] o exercício físico melhora muito porque antes tava com muita dor, se fizer um pouco há o alívio, é necessário o exercício físico para a melhora do dia a dia.

\section{Emocional}

[...] além do benefício do exercício físico em si, eu acho que tem a melhora da nossa auto-estima. 
[...] pegando um trem cheio pra caramba, aí chego aqui, a ginástica melhora, é o contato com as minhas colegas, é muito bom. Eu não diria que eu não me sinto tão bem fisicamente, mas mentalmente eu me sinto muito bem.

\section{Espiritual}

[...] eu acho que o sol representa a vida, é luz, desembaraço, claridade, energia, tudo de positivo que a gente pode ter na vida... eu também pensei no meu estado hoje de saúde, tá bom... tá como o sol: brilhando.

\section{Intelectual}

[...] aprendi a respirar, coisa que a gente não sabia, aprendi em casa a abaixar para pegar uma coisa embaixo da pia, quer dizer, tem a ver, porque melhorou muito a minha condição de saúde, das coisas que eu fazia errado também além da fibro."

Figura 1. Amostras aleatórias- relação entre exercício físico e saúde

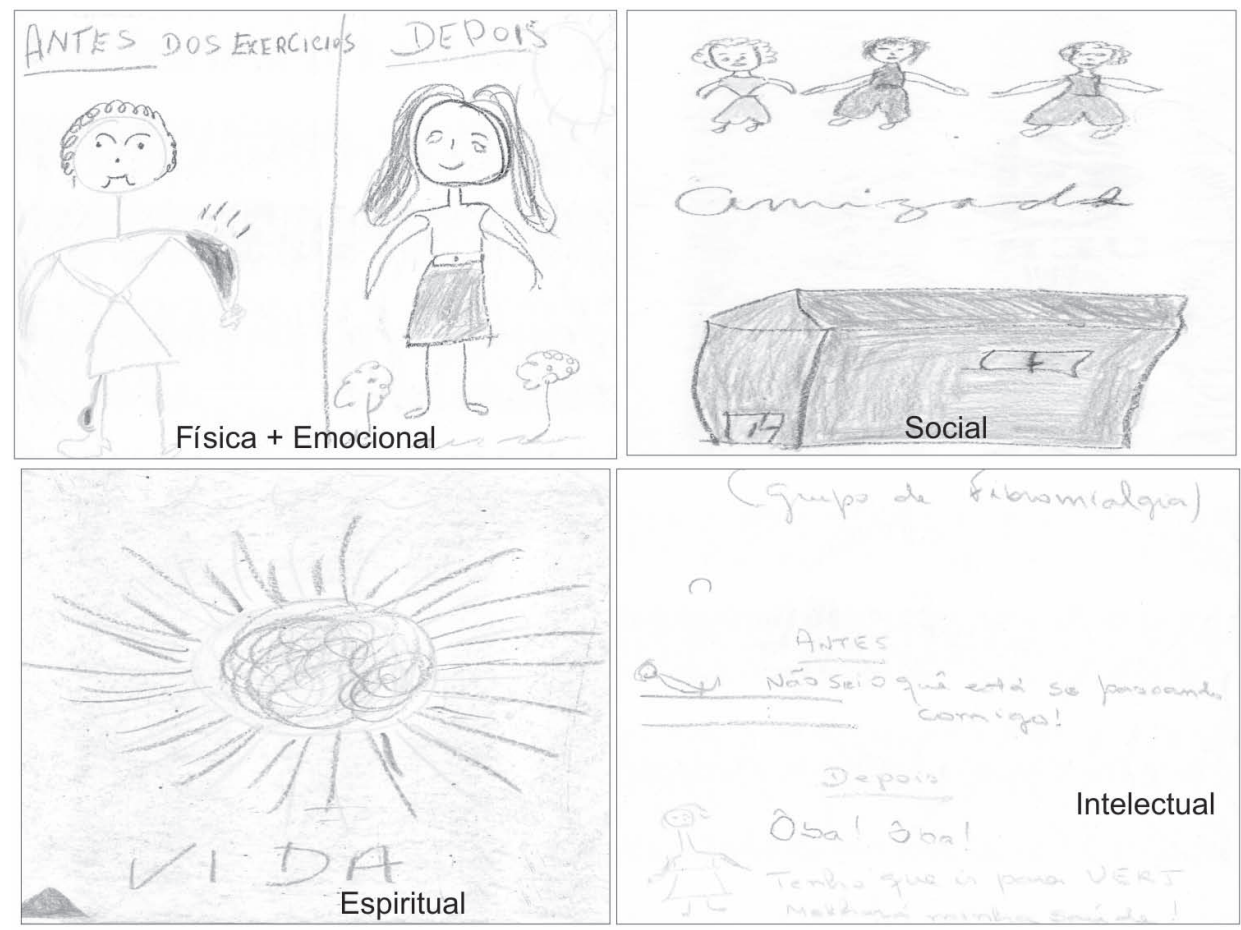


De acordo com Ibáñez apud Mazzotti (1994), o núcleo figurativo é a parte mais sólida e estável da representação e dele depende o significado desta. É o resultado das imagens e conceitos obtidos de modo a proporcionar uma imagem coerente e facilmente exprimível dos elementos que constituem o objeto da representação. Sendo assim, o núcleo figurativo identificado através das falas das participantes, para a relação exercício físico e saúde, foi a dimensão física expressa fundamentalmente a partir do alívio da dor, enquanto que o observado a partir das ilustraçōes foi a dimensão emocional expressa a partir da a melhora da auto-estima.

\section{Discussão}

Dentre as dimensões observadas na análise dos discursos, só a dimensão profissional não foi mencionada, o que pode ser explicado em função de se tratar de uma amostra onde apenas cinco mulheres desempenhavam atividades laborais.

Ao observar os resultados, a análise das falas ressaltou a dimensão física (100\%), através dos relatos não só de alívio da dor, como de melhor tolerância à dor. No que diz respeito à análise das falas referentes às ilustrações, a dimensão emocional foi a de maior representatividade (77,27\%), identificada através de melhora da autoestima, dados que não foram encontradas em nosso estudo (CAVALIERE, 2002).

Ressalta-se assim a relevância da abordagem qualitativa, que, através da teoria das Representações Sociais (JODELET, 2001), permitiu obter informações que podem auxiliar na avaliação da efetividade das ações propostas para o controle da síndrome. A pesquisa de representações oferece elementos e referências importantes para a construção do conhecimento da relação entre exercício físico e saúde, a partir de informações significativas das participantes deste estudo.

Cabe comentar que a hipertensão arterial sistêmica não aparece na fala do grupo, apesar de estar presente em mais de 50\% das participantes. A expressividade das dimensões física e emocional encontrada neste estudo talvez justifique este dado no que se refere à percepção dos diferentes sintomas da FM (dor generalizada) e HAS (na maior parte dos casos sem sintomas), o que sugere que essa enfermidade possa representar para participantes deste estudo um problema de saúde de menor impacto quando na presença da FM.

No que diz respeito à dimensão física da relação exercício físico e saúde, o exercício físico tem-se destacado como elemento relevante no tratamento da fibromialgia (BENNETT, 1996). Moldofsky (1975, 1976) observou que 
uma melhor aptidão cardiovascular pode melhorar alguns dos sintomas dessa síndrome. Estudos clássicos realizados por McCain (1986, 1988) revelaram benefícios do exercício físico, tais como, aumento da aptidão física e redução da dor à compressão dos pontos dolorosos. Mengshoel (1992) e Martin (1996) observaram que o exercício físico melhora a dor. Além da melhora da dor, Wigers et al. (1996), Gowans e Dehueck (1999) e Mannerkorpi et al. (2002) mostraram, em seus estudos, evidências de melhora do sono e da fadiga. Richards e Scott (2002) comentam também a melhora dos pontos dolorosos.

No que tange à dimensão emocional dessa relação, Benett (1996) e Gowans e Dehueck (1999) informam que pacientes com FM reconhecem a influência da prática regular de exercícios físicos no aumento da sensação de bem-estar. Segundo McCain $(1986,1988)$, essa mudança no estado emocional, junto com outras mudanças físicas, pode melhorar taxas de dor e escores psicológicos. Melhora de aspectos psicológicos também foram notadas por Ransay et al. (2000) e Assis et al. (2003). Wigers et al. (1996), Mannerkorpi et al. (2002) e Valim et al. (2003) observaram a melhora da depressão.

Considerando a dimensão intelectual, em 2001, Poyha et al. (2001) relataram que os pacientes com FM identificavam o exercício físico como intervenção não-medicamentosa de maior auxílio no controle da síndrome. Benett (1996) comenta sobre a importância da prática regular de exercícios físicos na aquisição de um novo estilo de vida

Em relação à dimensão social do objeto deste estudo, Schachter et al. (2003) informam a importância do grupo na adesão ao exercício físico em estudo sobre exercício físico em domicílio, mostrando elevadas taxas de evasão, o que pode comprometer a avaliação dos desfechos. Nichols e Glenn (1994) comentam que os encontros nas sessões de exercícios físicos podem propiciar o desenvolvimento de laços emocionais e sociais que contribuam para a melhora observada no grupo que se exercita. Benett (1996) reforça a importância do trabalho em grupo como motivador na aquisição de novas habilidades que aumentam a sensação de controle da situação.

Knoplich (2001) cita que valores morais, fatores sociais, forças internas (que quase todos acreditam), forças externas (que alguns duvidam) e influências divinas (fundamentais para muitos) podem influenciar na evolução de uma doença ou sintoma, de uma forma que a ciência ainda não sabe avaliar, ressaltando aspectos referentes à dimensão espiritual. 
A busca por artigos referentes a representações sociais e doenças crônicas que foi feita através da base de dados do Medline/PubMed e em outras fontes de consulta (HighWire), mostrou um número reduzido de publicações sobre o tema. Foi utilizada para busca a associação "social representation and chronic disease", desde o ano de 2000.

Quando considerada a especificidade da população estudada (FM), foi feita a busca nas mesmas bases de dados com a expressão "social representation and fibromyalgia”, desde o ano de 2000. Nenhuma publicação foi identificada, o que não permitiu a comparação dos resultados deste estudo com outros trabalhos. Este fato pode, por um lado, representar uma limitação, mas por outro destaca o aspecto original do trabalho.

Ainda na discussão sobre as limitações do estudo, o enfoque das representações sociais, cujos dados são obtidos a partir dos relatos e imagens dos participantes, confere, numa primeira análise, um grau de subjetividade. Entretanto, descrição objetiva dos elementos que compõem cada dimensão minimiza os diferentes entendimentos de cada categoria. Este fato pode ser identificado pela pequena margem de discrepância observada durante a categorização e análise dos dados, complementado pela exaustiva discussão até o consenso.

Considerando a FM como uma enfermidade crônica, a percepção da relação positiva entre exercício físico e saúde pode contribuir para a adesão ao tratamento e para o estabelecimento de uma melhor relação do indivíduo com a sua doença/ saúde. Para os profissionais envolvidos em programas de tratamento de pacientes portadores de FM, os resultados deste estudo reforçam a importância da visão holística do ser humano. É consenso, entre os pesquisadores da área, que as representações sociais, enquanto produtos sociais, têm sempre que ser remetidas às condições sociais que as engendraram - ou seja, o contexto de produção. Nesse sentido, destacamos o perfil do trabalho em grupo como um dos fatores que contribuíram para a formação das representaçôes encontradas, enfatizando, como já é bastante evidenciado na literatura científica, a importância da prática de exercícios físicos para a melhoria da qualidade de vida das pessoas.

Também é importante ressaltar que a análise qualitativa permitiu desvelar modificações positivas nas dimensões física e emocional, alterações que não tinham sido identificadas pela análise quantitativa como já citado anteriormente.

Podemos concluir que as participantes deste estudo reconhecem a relação positiva que existe entre o exercício físico e a saúde. 


\section{Referências}

ASSIS, M.R.; SILVA, L.E.; ALVES, A. et al. Deep water running: aquatic exercise to treat fibromyalgia: a randomized, controlled study. Arthritis Rheum, v. 48, p. S.303, 2003.

BARDIN, L. Análise de conteúdo. Lisboa: Edições 70, 2000. 225p.

BENNETT, R.M. Fibromyalgia and the disability dilemma - A new era in understanding a complex, multidimensional pain syndrome. Arthritis Rheum, v. 39, n. 10, p. 1634-37, 1996. . Multidisciplinary group programs to treat fibromyalgia patients. Rheum Dis Clin North Am, v. 22, p. 351-67, 1996.

BENNETT, R.M.; BURCKHARDT, C.S.; CLARK, S.R. et al. Group treatment of fibromyalgia: A 6-month outpatient program. J Rheumatol, v. 23, p. 521-8, 1996.

BENNETT, R.M.; CLARK, S. R.; GOLDBERG, L. et al. Aerobic fitness in patients with fibrositis. A controlled study of respiratory gas and exchangeand ${ }^{133}$ Xenon clearance from exercising muscle. Arthritis Rheum, v. 32, p. 454-60, 1989.

BUSCH, A.; BARBER, K.A.R.; OVEREND, T.J. et al. Exercise for treating fibromyalgia syndrome. The Cochrane Library. Oxford Update Software, n. 4, 2007.

CATHEY, M.A.; WOLFE, F.; KLEINHEKSE, S.M. et al. Socioeconomical impact of fibrositis. A study of 81 patients with primary fibrositis. Am J Med, v. 81, n. 3, S1, p. 78-84, 1986.

CATHEY, M.A. et al. Functional ability and work status in patients with fibromyalgia. Arthritis Care Res, v. 1, p. 85-98, 1988.

CAVAlCANTE, A.B.; SAUER, J.F.; CHALOT, S. D. et al. A prevalência da Fibromialgia:uma Revisão de Literatura. Rev Bras Reumatol, v. 46, n. 1, p. 40-48, 2006.

CAVAliERE, M.L.A. A Efetividade de um Programa Multidisciplinar de Tratamento para Fibromialgia. 2002. 87 p. Dissertação (Mestrado em Saúde Coletiva) - Instituto de Medicina Social, Universidade do Estado do Rio de Janeiro, Rio de Janeiro, 2002.

GOLDENBERG, D.L.; BURCKHARDT, C.; CROFFORD, L. Management of fibromyalgia syndrome. JAMA, v. 292, n. 19, p. 2388-2395, 2004.

GOWANS, S.E.; DEHUECK, A.; VOSS, A. et al. A randomized, controlled trial of exercise and education for individuals with fibromyalgia. Arthritis Care Res, v. 12, p. 120-8, 1999.

HAWLEY, D.J.; WOLFE, F.; CATHEY, M.A. Pain, functional ability and psychological status: A 12-month study of severity in fibromyalgia. J Rheumatol, v. 15, p. 1551-61, 1988.

HOLLMANN, W.; HETTINGER, T. Medicina do esporte: fundamentos anatômico-fisiológicos para prática esportiva. Barue: Manole, 2005. 710 p.

JODELET, D. As Representações Sociais: um domínio em expansão. In: (Org.). As Representações Sociais. Rio de Janeiro: Eduerj, 2001. p. 17-44.

JONES, K.D.; ADAMS, D.; STONE, K.W. et al. A comprehensive review of 46 exercise treatment studies in fibromyalgia. Health an quality of life outcomes, v. 4 n. 67, p. 1-6, 2006. 
KAPLAN, R.M.; SCHMDIT, S.M.; CRONAN, T.A. Quality of well being in patients with fibromyalgia. J Rheumatol, v. 27, n. 3, p. 785-789, 2000.

KNOPLICH, J. Desatando o nó do diagnóstico. In: . (Org.). Fibromialgia: dor e fadiga. São Paulo: Robe, 2001. p. 63-69.

MANNERKORPI, K.; BURCKHARDT, C.S.; BJELLE, A. Pool exercise combined with and education program for patients with fibromyalgia syndrome A prospective randomized study. Scand J Rheumatol, v. 31, p. 306-310, 2002.

MARTIN, L.; NUTTIG, A.; MACINTOSH, B.R. et al. An exercise program in the treatment of fibromyalgia. J Rheumatol, v. 23, p. 1050-3, 1996.

MARTINEZ, J. E. Avaliação da qualidade de vida de pacientes com fibromialgia através do "Medical Outcome Survey 36 Item Short Form Study". Rev Bras Reumatol, v. 11, n. 6, p. 312-316, 1999.

MAZZOTTI, A.J.A. Representações Sociais: aspectos teóricos e aplicações à Educação. Em aberto, v. 61, p. 60-78, 1994.

McARDLE, W.D.; KATCH, F.I.; KATCH, V.L. Fisiologia do exercício: energia, nutrição e desempenho humano. Rio de Janeiro: Guanabara Koogan. 2007. 1099 p.

MCCAIN, G.A. Role of physical fitness training in the fibrositis/fibromyalgia syndrome. Am J Med, v. 81, Suppl 3 A, p. 73-7, 1986,

MCCAIN, G.A.; BELL, D.A.; MAI, F.M. et al. A controlled study of the effects of a supervised cardiovascular fitness training program on the manifestations of primary fibromyalgia. Arthritis Rheum, v. 31, p. 1135-41, 1988.

MENGESHOEL, A.M.; FORRE, O.; KOMNAES, H.B. Muscle strength and aerobic capacity in primary fibromyalgia. Clin Exp Rheumatol, v. 8, p. 475-9, 1990.

MENGESHOEL, A.M.; KOMNAES, J.B.; FORRE, O. The effect of 20 weeks of physical fitness training in female patients with fibromyalgia. Clin Exp Rheumatol, v. 10, p. 345-9, 1992. MOLDOFSKY, H.; SCARISBRICK, P. Induction of neurasthenic musculoskeletal pain syndrome by selective sleep stage deprivation. Psychosom Med, v. 38, p. 35-44, 1976.

MOLDOFSKY, H.; SCARISBRICK, P.; ENGLAND, R. et al. Musculoskeletal symptoms and non-REM sleep disturbance in patients with "fibrositis syndrome" and healthy subjects. Psychosom Med, v. 37, p. 341-51, 1975.

NICHOLS, D.S.; GLENN, T.M. Effects of aerobic exercise on pain perception, affect, and level of disability in individuals with fibromialgia. Phys Ther, v. 74, p. 327-32, 1994.

PÖYHIÄ, R.; COSTA, D.; FITZCHARLES, M.A. Pain and pain relief in fibromyalgia patients followed for three years. Arthritis Care Res, n. 45, p. 355-61, 2001.

PROVENZA, J.R. et al. Diretrizes e bases para o diagnóstico e tratamento da fibromialgia. Rev Bras Reumatol, v. 44, p. 49-61, 2004. 
RANSAY, C.; MORELAND, J.; HO, M. et al. An observer-blinded comparison of supervised and unsupervised aerobic exercise regiments in fibromyalgia. Rheumatol, v. 39, p. 501-505, 2000.

RICHARDS, S.C.M.; SCOTT, D.L. Prescribed exercise in people with fibromyalgia parallel group randomized controlled trial. BMJ, v. 325, p. 185-187, 2002.

SCHACHTER, C.L.; BUSCH, A.J.; PELOSO, P.M. et al. Effects of short versus long bouts of aerobic exercise in sedentary women with fibromialgia: a randomized controlled trial. Phys Ter, v. 83, p. 340-58, 2003.

SENA, E.R.; DE BARROS, A. L.; SILVA, E.O. et al: Prevalence of rheumatic diseases in Brazil: a study using the COPCORD approach. J. Rheumatol, v. 31, p. 594-7, 2004.

SILVA, M.A.; DE MARCHI, R.D. Saúde e qualidade de vida no trabalho. São Paulo: Best Seller, 1997.

SOUZA FILHO, E.A. Análise de representações sociais. In: SPINK, MJP. O conhecimento no cotidiano: as representaçôes sociais na perspectiva da psicologia social. São Paulo: Brasiliense, 1995. p. 109-145.

SPINK, M.J.P. O estudo empírico das representações sociais. In: . (Org.). O conhecimento no cotidiano: as representações sociais na perspectiva da psicologia social. São Paulo: Brasiliense, 1995. p. 85-108.

VALIM, V.; OLIVEIRA, L.M., SUDA, A.L. et al. Aerobic fitness effects in fibromyalgia. J Rheumatol, v. 30, p. 1060-1069, 2003.

. et al. Peak oxigen uptake and ventilatory anaerobic threshold in fibromyalgia. $J$ Rheumatol, v. 29, n. 2, p. 353-357, 2002.

WIGERS, S.H.; STILES, T.C.; VOGEL, P.A. Effects of aerobic exercise versus stress management treatment in fibromyalgia: a 4.5 year prospective study. Scand J Rheumatol, v. 25, p. 77-86, 1996.

WOLFE, F.; SMYTHE, H.A.; YUNUS, M.B. et al. The American College of Rheumatology 1990 criteria for the classification of fibromyalgia. Arthritis Rheum, v.33, p. 160-72, 1990. WORLD HEALTH ORGANIZATION. Habitual physical activity and health. Cophenhagen: WHO Regional Office for Europe, 1978 (WHO Regional Publications, European Series, n.6).

\section{Notas}

${ }^{1}$ Consumo máximo de oxigênio: é o momento onde o consumo de oxigênio alcança um platô ou aumenta apenas levemente com os aumentos adicionais na intensidade do exercício (McARDLE; KATCH; KATCH, 2008, p. 170).

${ }^{2}$ Flexibilidade: é a amplitude de movimento arbitrariamente possível em uma ou em várias articulaçôes (HOLLMANN; HETTINGER, 2005, p. 152). 


\section{EFETIVIDADE DE UM PROGRAMA MULTIDISCIPLINAR NO TRATAMENTO DA FIBROMIALGIA}

\section{TERMO DE CONSENTIMENTO LIVRE E ESCLARECIDO}

Concordo voluntariamente em participar deste estudo cujo objetivo é avaliar os efeitos de um programa para tratamento da Fibromialgia. Tenho conhecimento de que a pesquisa inclui tratamento clínico acompanhado por médica reumatologista e psicóloga e treinamento físico ministrado por urna profissional de educação física.

De acordo com os procedimentos do estudo, estou informada que fui selecionada para participar de um programa de exercícios físicos, realizado duas vezes por semana e de sessōes quinzenais de psicoterapia em grupo além das consultas ambulatoriais. Sei também, que a duração prevista tanto para o programa de exercícios físicos quanto para psicoterapia é de seis meses

Fui informada que haverá avaliação clínica prévia da dor, depressão, capacidade física e flexibilidade das articulações e tendões além do meu estado global de saúde e condições para realização de tarefas do dia a dia, durante e ao final do período de estudo. Essas avaliações orientarão a equipe de pesquisa na prescrição dos tratamentos, incluindo os exercícios físicos.

De acordo com as exigências éticas de pesquisa médica, foi-me assegurado que as informaçôes individuais obtidas nas avaliações e nas sessões de treinamento físico terão caráter confidencial e apenas os membros da equipe de pesquisa terão acesso a elas. Sua utilização na pesquisa e na elaboração de relatórios e trabalhos científicos não identificará os pacientes participantes do estudo.

Estou esclarecida com relação aos possíveis desconfortos e riscos que podem ocorrer no início do treinamento físico e os procedimentos que serão utilizados para minimizá-los, tais como, a prescrição individualizada do treinamento e a progressão gradual da intensidade e duração do exercício físico. Será respeitada a minha opinião sobre a minha capacidade de fazer esforço físico. A equipe de pesquisa assegurou-me o direito de esclarecer qualquer dúvida que surgir a qualquer momento de realização da pesquisa, e também o direito de desistir de participar deste estudo a qualquer momento.

$\mathrm{Li}$, compreendi e concordo com os termos das declaraçôes acima prestadas.

Rio de Janeiro, de de 2001.

Paciente:

Testemunha:

Pesquisador: 
Representations of the relationship between physical exercise and health for patients with fibromyalgia

The fibromyalgia syndrome (FMS) is characterized by widespread chronic musculoskeletal pain, fatigue, disturbed sleep and associated symptoms. Its increasing prevalence and life quality impairment of patients justify researches for its control. Physical exercise (PE) is a relevant therapeutic tool among nonpharmachologic treatments options. It is important to mind the impact of FMS and different therapeutics on health, here understood as a set of dimensions: physical, emotional, social, spiritual, intellectual and professional. This study aimed to identify FMS patients' perception of relationship between PE and health. It used the theoretical methodological reference of Social Representation to study 22 women of a multidisciplinary project: Physical Education, Nutrition, Medicine and Psychology. Data analysis considered transcription of recorded semistructured interview, and interpretation of patients' illustrations. There were improvements in physical dimension evidenced by pain relief. Positive effects were also observed in emotional dimension, evidenced by self-esteem improvement, which appeared in the pictures. Based on the theory adopted, it was possible to identify that patients recognize a positive relationship between PE and health.

> Key words: treatment; fibromyalgia syndrome; social representation. 